\title{
Relationship between Urinary and Serum Level of Adiponectin with Disease Activity in Patients with Lupus in Comparison with Control Group S Sedighy ${ }^{1}$, M Aghaei $^{2}$, MA Vakili $^{3}$, M Fatemi $^{4}$, M faghani $^{5}$
}

\begin{abstract}
Objective: Systemic lupus erythematosus (SLE) is a connective tissue disease with chronic and recurrent and inflammatory progression. Antiinflammatory cytokines such as adiponectin change in this disease like other inflammatory diseases. This study aimed to evaluate the relationship between urinary and serum level of Adiponectin with disease activity in patients with SLE.

Methods and materials: In this case-control study, 80 women referred to the rheumatology clinic of 5 Azar Hospital in Gorgan, divided to case and control group. Then urinary and serum level of adiponectin measured by ELISA kit and disease activity evaluated by SLE disease activity index were Blood samples were taken from both groups and serum levels of interleukin -2 measured by AviBion human IL-2. Data analysis conducted by SPSS software (version 16) and by using descriptive statistics and statistical tests.

Results: Mean serum level of adiponectin in case group had statistically significant association with control group $(\mathrm{p}<0.001)$ and mean urinary adiponectin level in case group had statistically significant correlation with control group too $(\mathrm{p}<0.035)$. Only serum level of adiponectin was significantly associated with SLE disease activity index in case group $(r=0.63, p<0.0001)$ and urinary level of adiponectin was significantly associated with renal involvement $(r=0.59, p<$ 0/0001).
\end{abstract}

Conclusions: Findings of the present study showed the relationship between serum level of Adiponectin with disease activity in patients with SLE. So this biomarker can be used for effective treatment and decrease of complications.

From: ${ }^{1,2}$ Bone, Joint and Connective tissue disorder Research Center, sayyad shirazi hospital ,Gorgan, Iran, ${ }^{3}$ Department of Statistics, Medical School, Golestan University of Medical Sciences ,Gorgan, Iran and ${ }^{4,5}$ Clinical Student Research Committee, Faculty of Medicine, sayyad shirazi hospital ,Golestan University of Medical Sciences, Gorgan, Iran.

Correspondence: Sima Sedighy and maryam faghani, Golestan University of Medical Sciences, sayyad shirazi hospital, Gorgan, Iran. E-mail:simasedighy@yahoo.com or Sima_sedigh@yahoo.com 


\section{INTRODUCTION}

Systemic lupus erythematosus is, a connective tissue disease that is chronic, relapsing, and inflammatory .Involving of various Life support organs, such as kidney, central and peripheral nervous system, psychiatric and cardiovascular diseases in which occurs (1). Clinical features and mortality in this disease is due to tissue damage caused by the disease or side effects of cytotoxic drugs that is used for treatment of lupus (2). Although the aethiology of SLE is unknown, but there are many reasons show that, a combination of genetic, immunological, hormonal and probably "environmental factors have role in this disease (3).

Lupus erythematosus complications such as renal involvement, cardiovascular complications, hypertension and the need for immunosuppressive drugs Consumption, can affect on fertility, course of pregnancy, breast feeding and can be caused irreversible neonatal complications such as heart block. Thus, with regard to this entry, Importance of detecting markers of disease activity and its diagnosis become clear. Renal involvement in patients with SLE is one of the most common and dangerous complication of disease, that it has poor prognosis in majority of cases. The use of immunosuppressive drugs is an effective method in the management and improvement of it. Unfortunately, the use of these drugs increases the risk of morbidity (Infections, malignancies, metabolic disorders, infertility) and mortality in patients (4). Adiponectin is a $30 \mathrm{kDa}$ protein almost exclusively is constructed by adipose tissue [adipocytes] (5).

It has anti-inflammatory properties, and it increases sensitivity to insulin (6). It is similar to one of the components of the complement system [c1q] (7). This substance impact on inhibition phagocytic activity of macrophages and reduces production of TNFa. Also, it inhibits Monocyte production in the bone marrow and cell proliferation by inducing 
apoptosis $(8,9)$. Adiponectin, with a direct effect on endothelial cells increases production of nitrous oxide, so probably it has a vasodilatory role (10).

Its specified that in those People who have low adiponectin level vasodilatory mechanism of endothelial acts defective (11). This substance by reducing levels of gene expression like: (VCAM-1 (vascular cellular adhesion molecule, ICAM-1 (intra cellular adhesion molecule-1) and E-selectin, Block TNFa effects (12) recently, in the SLE patient monitoring of Changes in serum and urinary biomarkers, has been considered As a method ,for diagnosis of renal involvement and severity of disease.

Nevertheless, up to now valid and reliable biokmaker for this disease has not been determined. We, do this research on urinary and serum adiponectin levels in patients with SLE disease.

\section{SUBJECTS AND METHOD}

This Case control study was conducted on patients with SLE Patients that referred to Rheumatology clinics in the city of Gorgan per year by 2011-2012.

Patient Selection has been equally a non-randomized and available manner and among patient with definite SLE, according to the (ACR: American College of Rheumatolog criteria.

Based on M-Otero Study, the reference numbers 18, Volume of the samples were calculated 40 subjects In each group so (40 cases and 40 controls) were chosen.

The study group consisted of 40 women with SLE disease, and control group was made up of 40 women who were healthy, based on clinical examination, and results of tests as 
ANA, Anti DNA, UA, CBC, FBS and SLE disease was ruled out in them also they were similar to patients In term of age and weight.

Patients with END-stage renal disease (ESRD), pregnant adult females, patients with fasting glucose more than 90, patients with concurrent disease or other malignancies, and patients with other Rheumatologic diseases (syndromes Overlap) were excluded. (From the field) Sampling, was done from 9 am to 12 am With observance, of fasting. The time performing of this study, was since the beginning of September in 2011, until the beginning of February, in 2012 year. Data collection, was through interviews, examination and performing the necessary tests.

Serum and urine adiponectin tests, was conveyed by high sensitivity ELISA kit (High sensitive ELISA) for both the patients and control groups. Serologic tests, including $\mathrm{C} 3, \mathrm{C} 4$, and a collection of 24-hour urine, just performed in cases group, but ANA and Anti DNA test was done for both groups. After centrifugation of patient's blood, their serum, was held at a temperature of $-20^{\circ} \mathrm{C}$. After touching the samples to the required level, they were transported to laboratories outside the hospital for testing.

Urine samples (UA), was conducted in both groups in order to measure urinary adiponectin. Urine samples also kept at- 20 degree up to achieve the required level. Inclusion to study was totally voluntary and they could be omitted from the study at any time they want. All The obtained data were confidential. The identity of individuals not mentioned. At the time of reporting Project. data, imported into, computer and were analysed by using software SPSS16. To see the relationship between the variables, statistical test, including $t$-test, Chi-squared and Spearman's correlation coefficient and linear regression with $95 \%$ confidence intervals at a significance level of 5\% was used. 


\section{RESULT}

This study was performed on 40 female patients with SLE disease and 40 healthy women who had been related Lupus to them. The control group was matched with patient group for age and body mass index.

The mean age of both cases and control groups was, respectively, $31 / 98 \pm 10 / 4$ and $31 / 75 \pm 9 / 9$ also average of body mass index (BMI) in patients and control groups, was originally, $27 / 5 \pm 6 / 8$ and $27 / 7 \pm 7 / 8$. According to The independent $t$-test, these averages, was not associated with significant differences.

Comparison of serum and urinary adiponectin in both groups showed that, the mean and standard deviation of serum adiponectin levels was originally $43 / 67 \pm 14 / 9 v s$ 28/92 \pm $18 / 9$ in the patient and control group that was higher than in the patient group in comparison to control group and the estimated average rating was, respectively equivalent to 53 and 28 in these two groups that was associated with a significant difference $(p<0 / 0001)$.

The results showed that the average and standard deviation of urinary adiponectin in both cases and the control group, was respectively $2 / 06 \pm 5 / 13$ and $0 / 54 \pm 1 / 38$ and the average of rating in two groups was originally estimated 45 and 36 that was (associated) with statistically significant difference $/(p<0 / 035)$.

The average of ANA plasma levels of patients and the control group was, respectively $116 / 25 \pm 67 / 04$ and $(3 / 15 \pm 2 / 28)$ also AntiDNA plasma levels in patients and control group, calculated ordinarily, as 135/80 $\pm 146 / 3$ and 9/90 $\pm 11 / 54$. According to $t$ test, there have been, a statistically significant difference between the averages of both groups $(p<0 / 0001)$.

Among patients group, have been identified that four patients (10\%) have active disease (SLEDAI $>=10)$ and 36 patients have inactive disease. 
In evaluating the relationship of disease activity with urinary and serum adiponectin levels, it was determined that there was no significant linear correlation between urinary adiponectin and severity activity of disease. However, a significant linear relationship existed between serum adiponectin with the severity of disease activity $(p<0.0001)$ and $(\mathrm{r}$ $=0 / 63)$.

An evaluation of, the relationship between the severity of renal involvement (24-hour urinary protein) with serum and urinary adiponectin it was determined (identified) that there was no significant linear relationship between Adiponectin levels and the severity of renal involvement, However, a significant linear relationship between urinary adiponectin with the severity of renal involvement, existed $(p<0 / 0001$ and $r=0 / 59)$. A survey of the relationship between severity of renal involvement (24-hour urine protein) with disease activity based on The estimated Spearman correlation coefficient showed that there was no significant linear correlation between these two characteristics $(p>0.09)$ and $(\mathrm{r}=05 / 0)$.

\section{DISCUSSION}

Adipocytokines, which include leptin, Resistin, Visfatin and adiponectin is mostly produced by Adipose tissue via its endocrine functions. With a wide variety effects on, angiogenesis, hemostasis and immunity. Adipose tissue consists of adipocytes and macrophages, which constitute a lower percentage of that. These macrophages can produceuce several cytokines, such as TNF $\alpha$ and IL-6.

Obese patients, compared to lean subjects have a low-grade systemic inflammation that was accompanied by (1), increased CRP and IL-6 levels. 
The link between systemic inflammation and adipose tissue represents a potential contribution with regard to the inflammatory response, which may be partly explained by the production of inflammatory cytokines.

Actually up to $30 \%$ of circulating IL-6 produce by macrophages of adipose tissue, ( 2 adiposese tissue is a major source of circulating proinflammatory cytokines (13).

Adipokines effects on immunity and inflammation has been demonstrated. Patients with SLE, have high levels of circulating adiponectin and patients with high plasma adiponectin had a poor prognosis in lupus nephritis.

It has been evidenced that patients with classic chronic/autoimmune inflammatory diseases, have high level of serum adiponectin and it has pro-inflammatory action.

However, Adiponectin act as anti-inflammatory substance by increasing IL-6 or TNF $\alpha$ production, decreasing $T$-cell function, inhibiting phagocytosis.

Although high adiponectin levels could be a compensatory response of the organism against inflammation, but some scholars consider that this explanation is most irrational and point to the activation role of adiponectin in NFKB.

It has been proven that LMW adiponectin has an inhibitory role of endotoxin-induced secretion of IL-6 and to induce IL-10 production.

While MMW and HMW adiponectin has been shown that promute IL-8 synthesis and monocyte chemoattractant protein-1[MCP-1] (14)

Our results showed that plasma levels of adiponectin were higher in patients with SLE disease in comparison to control group, and this difference was statistically significant. In Sada and colleagues' study at the Okayama that was performed on 37 patients with SLE and 80 control group, serum adiponectin levels in patients were significantly higher than the control group $[p<0 / 01](15)$. 
Chang et al study in Atlanta on 109 patients with SLE and 78 healthy subjects, indicated that the plasma adiponectin levels were originally $22 \pm 15 / 3$ and $17 / 9 \pm 28 / 7$ in patients and healthy area of subjects. That was higher in the patient group with significant differences [ $p<0 / 003]$ (16). Also, the study of Rovin et al (2005) showed that there was a statistically significant difference between the average of serum adiponectin level, in SLE patients and control group [ $p=0 / 0005](12)$.

However, the Al M, et al study in 2009 that was conducted on 105 children with SLE disease and 77 healthy control groups. Results showed that the mean serum adiponectin level was $15 / 6 \pm 7 / 3$ in patients and $15 / 4 \pm 8 / 2$ in healthy subjects, which indicates the lack of significant association between serum adiponectin and the lupus disease (17).

The reason for this lack of correlation can be explained by, Effects of medications used to treat lupus disease and patient selection in silent stage or with mild disease activity.

Due to the autoimmune and inflammatory nature of rheumatoid arthritis disease, related studies performed on these patients were reviewed. This way in M Otero study the plasma levels of hormones produced by fat tissue (adiponectin, leptin, Resistin, Vysfdyn) were measured in both cases and control group (18). The results of this study showed that in cases group which included 31 patients with rheumatoid arthritis the mean plasma adiponectin levels were higher than the control group with significant differences. Also In the Senolt et al study, at 2006 (19) and Laurberg et al study at 2009 (20), that both of them (which) were performed, on patients with rheumatoid arthritis, similar results were obtained. 
In Rovin and colleagues' study that longitudinally designed, patient with lupus disease were divided in two group the first one include patient with renal involvement and relapse of lupus.

The second group revealed patient with relapse of disease without renal involvement the result of this study showed that serum level of adiponectin in patient with renal failure was higher than patient without renal flair that was associated with statistically significant difference $(p<0.01)$. Also Urinary adiponectin in patients, either before or after relapse of disease, was significantly higher than the control group (12).

In our study, as well as Rovin and colleagues' study, urinary adiponectin in the patient group was significantly higher than the control group. Also, In our study (research), urinary adiponectin, had significant correlation with 24-hour urine protein that was a marker for severity of renal involvement during of disease and This result, were similar to the results of the (Rovin) study.

On The other hand, in our study, there was no important association between serum adiponectin and 24-hour urine protein. Also in Rovin study, serum adiponectin, had no significant difference in both groups that was similar to our study [ $p=0.09]$ (12).

Yshen Studies and colleagues showed that in patients with proteinuria more than $150 \mathrm{mg}$ per day, urinary adiponectin levels are more than patients with proteinuria less than 150 mg. that are associated with significant differences (21). Studies on patients with diabetic nephropathy also have shown a significant correlation between urinary adiponectin and severity of proteinuria $(19,20)$.

About The correlation between the severity of disease activity (score) [that was determined by SLEDAI forms] with urinary and serum levels of adiponectin, results of our 
study, showed the mean severity of disease activity. Were estimated (5/75 $\pm 2 / 88)$ and only serum adiponectin had a significant linear relationship with disease activity score.

In Chung and colleagues studied on 109 patients with systemic lupus the mean of disease activity were estimated $(4 / 1 \pm 4)$. That there were no significant associated with serum adiponectin levels (14). Al M, et al study (2009) on the severity of lupus disease activity, in 105 paediatric patients indicated that the severity of disease activity was not significantly associated with serum adiponectin levels (15). The outcomes of these studies, about the relationship between the severity of disease activity with serum adiponectin was contrary to the results of our work.

Among the reasons for this difference can be pointed to the following: 1- There was probably of affect the dependent variables and environmental factors that their influences cannot be omitted such as: Patient race, geographic conditions and climate of the region where the sampling is done.

2-Being different of laboratory kits that are used to measure serum and urinary adiponectin levels have an important role (is especially important).

\section{CONCLUSION}

Our study showed that urinary adiponectin, has a direct correlation with 24-hour urine protein levels. Considering that kidney, is a vital organ and commonly be involved during a disease and increase rate of patients. Urinary adiponectin can be used as a marker to determine the severity of renal involvement for more aggressive treatment, in order to preserve renal function. On the other hand our study showed that serum levels of adiponectin are significantly associated with the severity of lupus disease activity. While this is not true in regard to urinary adiponectin. So serum levels of adiponectin can be used 
as a biomarker for effective treatments, in order to cut off the disease and reducing its complication in other organs. However, due to conflicting results exist in different studies on the relation of adiponectin with lupus disease activity score. The need for further studies in different countries and on different patient race require.

Table 1: Distribution of numerical variables in two groups

\begin{tabular}{lll}
\hline Variables & $\begin{array}{l}\text { Control group } \\
\text { Mean } \pm \text { SD }\end{array}$ & $\begin{array}{c}\text { Patients group } \\
\text { Mean } \pm \text { SD }\end{array}$ \\
\hline Age & $31 / 75 \pm 9 / 99$ & $31 / 98 \pm 10 / 45$ \\
weight & $68 / 78 \pm 15 / 9$ & $6 / 51 \pm 17$ \\
hight & $158 / 08 \pm 7 / 67$ & $157 / 90 \pm 8 / 88$ \\
ANA & $3 / 15 \pm 2 / 58$ & $116 / 14 \pm 67 / 04$ \\
Anti ds DNA & $9 / 9 \pm 11 / 54$ & $135 / 79 \pm 146 / 3$ \\
Serum Adiponectin & $28 / 92 \pm 18 / 92$ & $43 / 67 \pm 14 / 93$ \\
Urine Adiponectin & $\mathbf{0 / 5 4} \pm 1 / 38$ & $\mathbf{2 / 0 6} \pm 5 / 13$ \\
BMI & $\mathbf{2 7 / 7 7} \pm 7 / 8$ & $27 / 54 \pm 6 / 89$ \\
\hline
\end{tabular}




\section{REFERENCES}

1. Wallace DJ. What's New in the management of lupus since 2000 JCR: J Clin Rheumatol 2006; 12: 307-13.

2. Edwolthy SM.Clinical manifestation of systemic lupus erythematosus In: Harris E, Budd R, Cary S, et al. Kelley's text book of rheumstology. 7th Ed. Philadelphia: Saunders. 2005; 1105-1123.

3. Mccarty DJ, Manzi S, Medsger TA, Ramsey-Goldman R, Laporte RE, Kwoh CK. Incidence of systemic lupus erythematosus race and gender differences. Arthritis \& Rheumatism 2005; 38: 1260-70.

4. Maeda K, Okubo K, Shimomura I, Funahashi T, Matsuzawa Y, Matsubara K. CDNA cloning and expression of a novel adipose specific collagen-like factor, apM1 (AdiposeMost Abundant Gene Transcript). Biochem. Biophys. Res. Commu. 1996; 221: 286-9.

5. Yoda-Murakami M, Taniguchi M, Takahashi K, Kawamata S, Saito K, Choi-Miura NH, et al. A change in expression of GBP28/adiponectin in 
carbon tetrachloride-administrated mouse liver. Biochem Biophys Res Commun 2001; 285: 372-7.

6. Diez JJ, Iglesias P. The role of the novel adipocyte-derived hormone adiponectin in human disease. Eur J Endocrinol 2003; 148: 293-300.

7. Ouchi N, Kihara S, Arita Y, Maeda K, Kuriyama H, Okamoto Y et al. Novel modulator for endothelial adhesion molecules: adipocyte-derived plasma protein adiponectin. Circulation 1999; 100: 2473-6.

8. Kawanami D, Maemura K, Takeda N, Harada T, Nojiri T, Imai Y et al. Direct reciprocal effects of Resistin and adiponectin on vascular endothelial cells: a new insight into adipocytokine-endothelial cell interactions. Biochem. Biophys Res 2004; 314: 415-9.

9. Chen H, Montagnani M, Funahashi T, Shimomura I, Quon MJ. Adiponectin stimulates production of nitric oxide in vascular endothelial cells. jbc. 2003; 278: 45021-6.

10. Tan K, Xu A, Chow W, Lam M, Ai V, Tam S, et al. Hypoadiponectinemia is associated with impaired endothelium-dependent vasodilation. J Clin Endocrinol Metab 2004; 89: 765-9.

11. Hu E, Liang P, Spiegelman BM. AdipoQ is a novel adipose-specific gene dysregulated in obesity. jbc. 1996; 271: 10697-703.

12. Rovin BH, Song H, Birmingham DJ, Hebert LA, Yu CY, Nagaraja HN. Urine chemokines as biomarkers of human systemic lupus erythematosus activity. J Am Soc Nephrol 2005; 16: 467-73. 
13. Toussirot É, Gaugler B, Bouhaddi M, Nguyen N. U, Saas P, Dumoulin G. Elevated adiponectin serum levels in women with systemic autoimmune diseases. Mediators Inflamm.2010; 938408. Published online Dec 26, 2010. doi: $\underline{10.1155 / 2010 / 938408}$.

14. Krysiak R, Handzlik-Orlik, G,Okopien B. The role of adipokines in connective tissue diseases.Eur J Nutr, 2012, 51.5: 513-528.

15. Sada KE, Yamasaki Y, Maruyama M, Sugiyama H, Yamamura M,Maeshima Y, et al. Altered levels of adipocytokines in association with insulin resistance in patients with systemic lupus erythematosus. J Rheumatol. 2006; 33: 1545-52.

16. Chung CP, Long AG, Solus JF, Rho YH, Oeser A, Raggi P, et al. Adipocytokines in systemic lupus erythematosus: relationship to inflammation, insulin resistance and coronary atherosclerosis. Lupus. 2009;18:799-806.

17. Al M, Ng L, Tyrrell P, Bargman J, Bradley T, Silverman E.Adipokines as novel biomarkers in paediatric systemic lupuserythematosus. Rheumatology (Oxford) 2009; 48: 497-501.

18. Otero M, Lago R, Gomez R, Lago F, Dieguez C, Gomez-Reino J, et al. Changes in plasma levels of fat-derived hormone adiponectin, leptin, 
Resistin and Visfatin in patients with rheumatoid arthritis. Ann Rheum Dis 2006; 65: 1198-201.

19. Senolt L, Pavelka K, Housa D, Haluzik M. Increased adiponectin is negatively linked to the local infl ammatory process in patients with rheumatoid arthritis. Cytokine 2006; 35: 247-52.

20. Laurberg TB, Frystyk J, Ellingsen T, Hansen IT, Jorgensen A, Tarp U et al. Plasma adiponectin in patients with active, early, and chronic rheumatoid arthritis who are steroid- and disease-modifying antirheumatic drug-naive compared with patients with osteoarthritis and controls. J Rheumatol 2009; 36: 1885-91.

21. Shen YY, Hughes JT, Charlesworth JA, Kelly JJ, Peake PW. Adiponectin is present in the urine in its native conformation, and specifically reduces the secretion of MCP-1 by proximal tubular cells. Nephrology .2008;13(5): 